\title{
LEBENSWELT UND INTENTIONALITÄT DER URTEILSBILDUNG: ZUR REVISION EINES PHÄNOMENOLOGISCHEN GRUNDKONZEPTS
}

JAGNA BRUDZIŃSKA

\begin{abstract}
Lifeworld and intentionality of judgment: For the revision of a phenomenological foundational concept
\end{abstract}

This paper approaches the notion of lifeworld as the original ground for intentional judgments. Husserl's phenomenology begins with a purely theoretical understanding of intentionality and judgment, but his position later evolves into a broader account of the practical and genetic, preverbal, and pre-reflective aspects of intentionality. This broadening of the conception of intentionality corresponds with the discovery of the complex, intertwined, and multi-layered dimension of lifeworld. An important step in the development of a more encompassing theory of lifeworld is provided by Husserl's reflection on Avenarius' notion of "natural world" already in 1910/11. However, Husserl reaches the full accomplishment of the theory of lifeworld and of the formation of judgment only by designing the phenomenological genetic method, thereby developing a new understanding of transcendental subjectivity and introducing the notion of type as a concrete generality arising from the lifeworld.

\section{Welt und Lebenswelt}

Die Lebenswelt als Boden der Erfahrung und Intentionalität als grundlegende Erfahrungsstruktur haben in der Phänomenologie nicht von Anfang an etwas miteinander zu tun. Die Phänomenologie beginnt mit der These der Intentionalität, begriffen als Aktivität des theoretischen Bewusstseinsaktes. Jegliche Gebundenheit des Bewusstseins, ob empirisch-psychologisch oder (lebens-)weltlich, wird hier 
dezidiert aus der Betrachtung ausgeschlossen. Weder wissenschaftliche Theorien noch individuelle Überzeugungen der in der Welt lebenden Personen - oder gar ihre körperliche Verfassung, ihre Gefühle, Wünsche, Sorgen und Ängste -, noch die Geschichte ihrer Erfahrungen und ihre soziale Verortung spielen eine Rolle, wenn es um die Aufklärung der intentionalen Urteilsleistung des Bewusstseins geht.

Husserl hält zwar fest, dass jedes Denkerlebnis und damit jede Urteilsleistung, ähnlich wie jedes psychische Erlebnis, nicht nur seinen deskriptiven Gehalt, sondern auch „in kausaler Hinsicht seine Ursachen und Folgen [hat], es greift irgendwie in das Getriebe des Lebens hinein und übt seine genetischen Funktio-

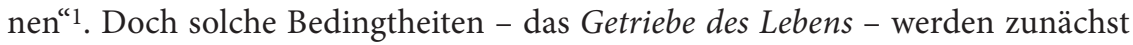
als außerwesentlich aus der Betrachtung ausgeschlossen. Das, was die deskriptive Phänomenologie interessiert „muß ausschließlich im ,Inhalt des Bedeutungs- und Erfüllungserlebnisses selbst', und zwar als Wesentliches aufgewiesen werden“2.

Das Motiv der Lebenswelt beginnt sich erst zehn Jahre später abzuzeichnen. Es sind die Vorlesungen zu den Grundproblemen der Phänomenologie aus dem Jahr 1910/11, in denen Husserl, in der Auseinandersetzung mit Richard Avenarius' empiriokritizistischer Position, die „natürliche Einstellung“ und mit ihr die „natürliche Erfahrung" als die Einstellung der lebensweltlichen Erfahrung entdeckt.

Avenarius geht in seiner Erfahrungsanalyse vom „unmittelbar Vorgefundenen“ aus. Nicht metaphysische Spekulation, sondern das, was unmittelbar und zweifelsfrei in der Erfahrung gegeben ist, markiert den Ausgangspunkt der philosophischen Analyse. Am Anfang allen Philosophierens steht der „natürliche Weltbegriff", und das bedeutet: Am Anfang stehe ich, meine Gedanken und Gefühle, mein Körper und meine Umgebung. ${ }^{3}$ Diese These greift Husserl in seinem neuen Erfahrungsstudium auf. Im Einklang mit Avenarius macht er den „natürlichen Weltbegriff“ zum Ausgangspunkt der Erkenntnistheorie, um von dort aus die Möglichkeit einer „erfahrenden Phänomenologie, die nicht Wesenslehre ist“ zu prüfen. ${ }^{4}$ Diese frühe „erfahrende Phänomenologie“ wird in den 30er Jahren zur Phänomenologie der Lebenswelt.

Husserl Edmund, Logische Untersuchungen, Untersuchungen zur Phänomenologie und Theorie der Erkenntnis, Zweiter Band - I. Teil, Edmund Husserl - Gesammelte Werke Husserliana Bd. XIII (= Hua XIX/1), Den Haag, Nijhoff, 1984, S. 150. (Alle Bände aus der Edition Husserliana werden im Folgenden mit der Abkürzung ,Hua, Bd. Nr. römisch` zitiert.)

Idem.

Avenarius Richard, Der menschliche Weltbegriff, Leipzig, Reisland, 1891, Abschnitt I.

4 Husserl E., Zur Phänomenologie der Intersubjektivität. Texte aus dem Nachlass. Erster Teil. 19051920, Hrsg. Hua XIII, I. Kern, Den Haag, 1973, S. 111, Anm. 1. 
In seiner in den Grundproblemen entwickelten Interpretation deutet Husserl den natürlichen Weltbegriff als Bereich unmittelbarer "Vorgefundenheiten" des erfahrenden Subjekts, die jeder theoretisierenden, damit wissenschaftlichen Auffassung vorausgehen. Es ist die Welt, die in der hier erstmals so genannten „natürlichen Einstellung" erfahren wird:

Was da unter dem Titel „vorfinden“ bezeichnet wurde und vor allem schließenden, geschweige denn wissenschaftlichen Denken liegt, ist nichts anderes als was auch im prägnanten Sinn erfahren heisst. Die natürliche Einstellung ist also die der Erfahrung. Das Ich erfährt sich selbst und macht Erfahrung über Dinge, über Leiber und fremde Ich. Diese Einstellung der Erfahrung ist die natürliche, sofern sie die ausschließliche ist des Tieres und des vorwissenschaftlichen Menschen. ${ }^{5}$

Die natürliche Einstellung ist also die Einstellung, in der wir als empirische Personen in der real existierenden Welt uns selbst vorfinden. Wir finden uns selbst als solche, die bestimmte Erlebnisse, Gewohnheiten, einen bestimmten Charakter und einen Körper haben, als solche ferner, die Erinnerungen und Erwartungen haben, uns körperlich immer in einer räumlichen Umgebung befinden, in der Zeit dauern, eine persönliche Geschichte, eine Biographie und Beziehungen mit anderen haben, die wir in unserer Welt ebenfalls vorfinden. Unsere körperliche Verfassung bindet uns dabei an die Natur. Letztere, die Natur, wird aber als ein von uns unabhängiges System raum-zeitlicher und kausaler Regelmäßigkeiten angenommen. Als solches gibt sie den Rahmen der wissenschaftlichen Reflexion. Die wissenschaftlichen Urteile basieren auf Erfahrungen in der natürlichen Welt. In dem Punkt ist sich Husserl mit Avenarius einig: Indem wir das in der natürlichen Einstellung Vorgefundene beschreiben, bilden wir Urteile, bis hin zu wissenschaftlichen Urteilen. Nun fordert Husserl eine Aufklärung jenes Urteilens, um die tieferliegenden Voraussetzungen der Urteilstätigkeit selbst aufzudecken, als es der Positivismus oder Empiriokritizismus beansprucht. Nach Husserls Diagnose sind letztere blind gegenüber der Daseinsprätention und so auch der Prätention der Naturthesis im natürlichen Welterleben und bleiben daher „im Naturalismus hängen“" ${ }^{\text {" }}$ Die Voraussetzung der Naturthesis soll nun im Vollzug der neu konzipierten reinen phänomenologischen Reduktion offengelegt und schließlich auch überwunden werden. ${ }^{7}$

\footnotetext{
Hua XIII, 120.

Vgl. Hua XIII, $199 \mathrm{f}$.

Vgl. Hua XIII, $138 \mathrm{ff}$. Hinsichtlich weiterer Aspekte von Husserls Auseinandersetzung mit Avenarius sowie der Philosophie des Positivismus verweise ich vor allem auf Sommer Manfred, Husserl
} 
Die reine phänomenologische Reduktion, die Husserl in den Grundproblemen entwickelt, erschließt ein neues Feld der transzendentalen Erfahrung. Die Reduktion wird hier nicht auf einen isolierten intentionalen Akt angewandt, wie es 1905 die Vorform der transzendentalen Reduktion in den Seefelder Blätter ${ }^{8}$ anstrebte oder die 1907 in Die Idee der Phänomenologie beschriebene transzendentale Reduktion im Fokus hatte. Vielmehr fordert Husserl von uns eine Änderung unserer gesamten Erfahrungseinstellung. Bildet die natürliche Einstellung, die eine ganze Welt zu ihrem Thema hat, den Ausgangspunkt der Erkenntniserfahrung, wird eine andere Einstellung notwendig, um die Voraussetzungen der Ausgangseinstellung zu durchleuchten. Wir treten einen Schritt zurück, wir lassen die Daseinsthesis und somit auch die Naturthesis fallen oder klammern sie ein. Die natürliche Welt verschwindet damit aber nicht, sondern wird unter veränderten Vorzeichen - wie Husserl dazu sagt - neu gewonnen. Ein unendlicher Bewusstseinsstrom, in dem sich jeglicher Sinn des in der natürlichen Welt zuvor Vorgefundenen konstituiert, wird zum neuartigen Erfahrungsfeld der phänomenologischen Empirie. Nicht das Was des Erscheinens, wie bei der Wesensforschung der früheren Aktphänomenologie, sondern das Wie des Erscheinens in seiner zeitlichen Extension und Dynamik wird zum Thema. Hier, betont Husserl, erscheint zum ersten Mal die Idee einer apodiktischen Kritik der phänomenologischen Erfahrung. ${ }^{9}$ Jene Kritik umfasst alle Gestalten der Gegebenheitsweisen der Erfahrung, d. h. Wahrnehmung, Retention, Wiedererinnerung, Erwartung und auch Einfühlung. Husserl konstatiert damit eine gewaltige Erweiterung des Erfahrungsfeldes. Alle Formen der Gegenwärtigung und Vergegenwärtigung bieten originäre Quellen phänomenologischer Erkenntnis. In dieser Schrift wird also die natürliche Welt als Vorläufer der Lebenswelt auf eine radikale Weise hinsichtlich des Wie ihres Erscheinens befragt. Erschlossen wird dabei ein neuartiges Feld der Subjektivität: ein zusammenhängender, motivational struk-

und der frühe Positivismus, Philosophische Abhandlungen Bd. 53, Frankfurt a./M., Vittorio Klostermann, 1985.

8 Ein Großteil der sogenannten Seefelder Blätter wurde als Text Nr. 35 in Hua X (Husserl E., Zur Phänomenologie des inneren Zeitbewusstseins (1893-1917). Hrsg. R. Boehm, Den Haag, Nijhoff, 1966) veröffentlicht. Ihre Bedeutung hinsichtlich der Entwicklung der Konzeption der transzendentalen Reduktion wird hervorgehoben von: Biemel Walter, „Einleitung des Herausgebers“, in Husserl E., Die Idee der Phänomenologie. Fünf Vorlesungen, Hua II, Den Haag, Nijhoff, 1958 VIII ff.; Melle Ullrich, „Einleitung des Herausgebers”, in Husserl E., Einleitung in die Logik und Erkenntnistheorie. Vorlesungen 1906/07, Hua XXIV, Den Haag, Nijhoff, 1984, XXI ff.; Bernet Rudolf, „Einleitung des Herausgebers", in Husserl E., Texte zur Phänomenologie des inneren Zeitbewusstseins (1983-1917). Hamburg, Meiner, 1985, XXXI ff.; Luft Sebastian, „Einleitung des Herausgebers”, in Husserl E., Zur phänomenologischen Reduktion. Texte aus dem Nachlass (1926-1935), Hua XXXIV, Dordrecht, Kluwer, 2002, Anm. 1.

9 Vgl. Hua XIII, 159 Anm. 1. 
turierter Bewusstseinsstrom. Allerdings wird hier keine ichliche Subjektivität postuliert. Die egologische Wende wird jedoch bald darauf, in den Ideen I und II, vollzogen. Sie ordnet diese transzendentale Welt-Erfahrung einem transzendentalen Subjekt zu. Dennoch haben wir es hier mit einem von Grund auf neuen Begriff der Subjektivität zu tun. Es ist eine personale weltvolle Subjektivität, die in den 30er Jahren auch explizit mit dem Terminus lebensweltliche Subjektivität ${ }^{10}$ bezeichnet wird.

Die weitere Annäherung beider Konzepte, des Konzepts der Intentionalität und der Lebenswelt, hängt also auch mit dem Wandel im Verständnis der intentionalen Subjektivität und der damit korrespondierenden Entwicklung der intentional-genetischen Fragestellung zusammen. Nicht einfach die Lebenswelt, sondern eine lebensweltlich bestimmte Subjektivität, eine personale, zugleich transzendental zu erforschende Person in ihrer Entwicklung und in der Konkretion ihrer Erfahrung wird zum Thema der phänomenologischen Forschung.

\section{Die Geschichte des Ich und die Intentionalität}

Der deskriptiven Aktphänomenologie geht es um die Konstitution von einheitlichen Gegenständen, die im Hinblick auf die zeitlose Struktur ihrer Identität beschrieben werden sollen. Das intentionale Erlebnis wird hierbei als quasi geschichtslose Vorfindlichkeit der intentionalen Erfahrung beschrieben. Die Deskriptionen zielen dabei nicht auf beliebige Eigenschaften des intentionalen Aktes, sondern auf seine Wesensstruktur, das Invariante oder das Essentielle des Erlebnisses. ${ }^{11}$ Das zu betrachtende Erlebnis als Untersuchungsgegenstand scheint jedoch im Zugriff der aktphänomenologischen Wesensforschung in einem gewissen historischen und situationellen Vakuum zu stehen. Es wird aus seinem lebendigen Zusammenhang herausgerissen, um einer nüchternen Analyse seiner Strukturmomente unterzogen zu werden. Es werden keine Motivationen der Auffassungsaktivität berücksichtigt, ebenso keine Dynamiken der Aufmerksamkeitsweckung, der Zuwendung etc. Auch wenn an dem Erlebnis leiblich kinästhetische oder affektive Momente erfasst werden, werden diese unter Abstraktion von dem lebendigen und dynamischen Entwicklungszusammenhang der intentionalen Erfahrung betrachtet. ${ }^{12}$

10 Husserl E., Die Krisis der europäischen Wissenschaften und die transzendentale Phänomenologie, Hua VI, Hrsg. W. Biemel, Den Haag, Nijhoff, 1954, S. 129; 223.

11 Vgl. Husserl E., Zur Lehre vom Wesen und zur Methode der Eidetischen Variation. Texte aus dem Nachlass (1891-1935), Hrsg. D. Fonfara, Hua XLI, Dordrecht, Springer, 2012, $29 \mathrm{ff}$.

12 Vgl. Husserl E., Ding und Raum. Vorlesungen 1907, Hrsg. U. Claesges, Hua XVI, Den Haag, Nijnoff, 1973, $164 \mathrm{ff}$. 
Die genannte Erweiterung des phänomenologischen Forschungsbereichs in den Grundproblemen der Phänomenologie impliziert dagegen eine gewichtige Differenzierung der Evidenz- und Vollzugsarten der Erkenntnis. Neben dem Vergangenheitsbewusstsein wird hier auch das Fremd- und das Zukunftsbewusstsein sowie der komplexe Verweisungszusammenhang der Erlebnisse zur primären Quelle der phänomenologischen Erfahrung als intentionaler Werdensprozess erläutert. Dadurch wird ersichtlich, dass, wie Husserl ausführt,

phänomenologische Erfahrung nicht an den vereinzelten cogitationes hängt, die jetzt beachtete Gegenwärtigkeiten sind, sondern [...] über den ganzen Strom des Bewusstseins als einen einzigen Zeitzusammenhang sich erstreckt, der freilich nicht in seiner ganzen Breite und Länge jeweils in das Licht der Anschauung fällt. ${ }^{13}$

Entscheidend dabei ist, dass der Bewusstseinsstrom als Zeitzusammenhang unter einer Gesetzlichkeit steht, die eine neue Methode ihrer Erforschung verlangt. Es ist die Methode der Motivationsanalyse. Denn es ist die Motivation, die hier die Dignität des intentional-genetischen Gesetzes im Aufbau der lebendigen Erfahrung erlangt:

Und da ist es eine Erkenntnis von ungeheurer Wichtigkeit, dass jede natürliche Erfahrung als immanentes Sein genommen eine Mannigfaltigkeit anderer natürlicher Erfahrungen und eine Mannigfaltigkeit von realen Möglichkeiten natürlicher Erfahrung motiviert und dass wir diese Motivationszusammenhänge, welche Zusammenhänge des reinen Bewusstseins sind, aufwickeln und auf sie unseren Blick richten können. Und dieser Blick hat den Charakter phänomenologischer Erfahrung. ${ }^{14}$

Die subjektive Realität wird dabei als dynamischer Zeitfluss des Erlebens erschlossen, als strömende Gegenwart, die sowohl Vergangenheit als auch Zukunft umfasst, Wahrnehmen und Phantasieren, Erinnern und Erwarten, Selbst- und Fremderleben in seinen leiblichen und seelischen, praktischen und theoretischen Zügen. Der Fluss des Bewusstseins, das Strömen des Erlebens, so wie es sich uns in der erlebten Innenerfahrung zeigt, vor jeder wissenschaftlichen Deutung und Erklärung, Bewertung und Auswertung, vor jeglicher Zurückführung auf physisch-kausale, naturalisierende oder metaphysisch-spekulative Annahmen, wird in seinen unterschiedlichen Gegebenheitsweisen als ein erfahrbares, unendliches Feld des subjektiven Lebens und somit des intentionalen Leistens ausgelegt, und

13 Hua XIII, $176 f$.

14 Hua XIII, 180; vgl. auch Hua XIII 78, 166, $176 f$. 
zwar als ein zeitlich-geschichtliches, motivationsgeleitetes und relationales Feld der genetischen Analyse. Das subjektive Leben ist von nun an ein Leben in die Geschichte und aus der Geschichte heraus. ${ }^{15}$

Aus Husserls transzendental-phänomenologischer und genetischer Perspektive erklärt sich die Identität der Erlebnisse nicht mehr allein aus der zeitlos gefassten Erlebnisstruktur, sondern aus der Geschichte ihres Entstehens. Und diese Geschichte bildet den Motivationshorizont jeglicher weiteren Erfahrung und intentionalen Sinn- und Urteilsbildung. Ein Erlebniszusammenhang oder ein einzelnes Erlebnis werden beide nur im Lichte ihrer konkreten geschichtlichen Individuation verständlich - einer Individuation, die sich auf dem Boden der Lebenswelt vollzieht:

Es kommen dann alsbald in Frage die anderen intentionalen Verweisungen, die zur Situation gehören, in der z. B. der die urteilende Aktivität Übende steht, also mit in Frage die immanente Einheit der Zeitlichkeit des Lebens, das in ihr seine „Geschichte“ hat, derart dass dabei jedes einzelne Bewusstseinserlebnis als zeitlich auftretendes seine eigene „Geschichte", d. i. seine zeitliche Genesis hat. ${ }^{16}$

Die Aufgabe der phänomenologischen Analyse besteht dabei in der Enthüllung intentionaler Implikationen und in der Rekonstruktion der Verläufe der Sinnbereicherung im Individuationsprozess. Im Zuge dessen werden die verborgenen Sinnesmomente offengelegt und es wird der Sinnes-Sedimentierung nachgegangen. Hierbei kommt es nicht nur auf bloße oder ursprüngliche Passivität an, sondern auch auf das, was Husserl als sekundäre Passivität bezeichnet: eine Passivität, die aus der Geschichte der aktiven Leistungen des lebensweltlichen Subjekts resultiert. ${ }^{17}$ Sie entsteht im Übergang von Aktivität zu Passivität des Bewusstseinsverlaufs und macht dabei die Unterlage unserer Bereitschaft zu weiteren konkreten

15 Mit dieser Einsicht ruckt Husserls transzendentale Phanomenologie deutlich in die Nahe der lebensphilosophischen Tradition. Dabei ist vor allem an Wilhelm Dilthey zu denken und sein Verstandnis der Geschichtlichkeit der menschlichen Person. Andererseits aber werden wir auch auf die philosophisch-anthropologischen Reflexionen Helmuth Plessners verwiesen, der im Anschluss an Dilthey den Menschen explizit in einer Doppelbestimmung gedeutet hat. Zum einen hat er in ihm das Subjekt der Geschichte gesehen, den Schöpfer der geschichtlichen Erfahrung und Deutung. Zum anderen erkannte er die menschliche Bedingtheit durch die Geschichte. Auch das Schaffen und Gestalten der Geschichte selbst ist also in Plessners Augen geschichtsbedingt. Der Mensch als solcher ist geschichtsbedingt und geschichtsbedingend. (Plessner Helmuth, Macht und menschliche Natur, Berlin, Suhrkamp, 2003, 185 ff).

16 Hua XVII, 316.

17 Vgl. z.B. Husserl E., Ideen zu einer reinen Phänomenologie und phänomenologischen Philosophie. Zweites Buch: Phänomenologische Untersuchungen zur Konstitution, Hua IV, Hrsg. W. Biemel, Den Haag, Nijhoff, 1952, 12. 
intentionalen Leistungen der Sinnkonstitution und Urteilsbildung aus. Husserl entwickelt in diesem Zusammenhang ein Interesse für die Bestimmung konkreter Motivationen unserer intentionalen Auffassungsleistungen.

\section{Die Genese der Urteile}

Ab dem zweiten Buch der Ideen (1913-16) wird der genetische Prozess als Ichwerdungsprozess nicht mehr als ein empirischer und somit unter kausalen Bedingtheiten des mundanen Subjekts stehender Verlauf verstanden. Vielmehr wird der immanente Motivationszusammenhang als Gesetzlichkeit des ichlichen Bewusstseinsstroms thematisch. ${ }^{18}$ Das ichliche Erlebnis erklärt sich dabei nicht allein aus seiner Struktur. Vielmehr wird es als in die subjektive Erfahrungsgeschichte eingebettet gesehen und nur aus dieser Geschichte heraus verständlich gemacht. Werdensprozesse und Individuationsdynamiken der egologischen Erfahrung werden hier in ihrer Horizonthaftigkeit ausgelegt. Sie werden im Hinblick auf ihre immanente Teleologie, also ihre immanente Zielgebundenheit, hin untersucht. Der subjektive Motivationsprozess soll offengelegt werden. Die Subjektivität wird auf die Strukturen und Dynamiken ihrer immanenten Zeitlichkeit hin erforscht. Dabei wird nicht nur die Vergangenheit, also die immanente Geschichte erforscht. Wie bereits die Bernauer Manuskripte (1917/18) gezeigt haben, ist neben der Vergangenheit auch die Zukunftsdimension von entscheidender Bedeutung für die Klärung der intentionalen Leistung. ${ }^{19}$ Eine neue dynamische Beziehung zwischen Vergangenheit und Zukunft wird aufgedeckt und als Leistungsstruktur der Erwartung im ichlichen Leben wiedergefunden. Erwartungen, ihre Bestätigungen und Enttäuschungen machen uns Personen als egologische Subjekte der Motivation verständlich. Es ist nicht die Zusammenstellung von personalen Dispositionen und Eigenschaften, die diese Verständlichkeit gewährleistet. Die Motivation ist auch dasjenige, das die Verständlichkeit der intentionalen Leistung der Personen trägt.

In seinem Studium der Bewusstseinspassivität in den 20er Jahren widmet sich Husserl der vorreflexiven intentionalen Synthesen der Assoziation, die nicht mehr bloß formal und damit empiristisch, sondern als inhaltliche motivationale Gesetzlichkeit verstanden wird. Neben der Assoziation widmet Husserl sich intensiv der Untersuchung der Affektion als elementare prä-intentionale Leistung. Sie wird

18 Hua IV, $211 \mathrm{ff}$.

19 Husserl E., Die Bernauer Manuskripte über das Zeitbewusstsein (1917/18), Hrsg. R. Bernet und D. Lohmar, Hua XXXIII, Dordrecht, Kluwer, 2001, 8 ff. 
nicht als ein abstraktes Moment der Reizbarkeit des Subjekts abgetan oder als eine bloß natürliche Reaktion des empirischen Ich auf äußere oder innere Reize verstanden. Husserl zeigt, dass es sich bei Affektionen immer um sinnvolle Antworten handelt, die ein lebensweltliches Subjekt charakterisieren. ${ }^{20}$ Diese elementaren, auch als prä-intentionale zu fassenden Leistungen werden noch differenzierter betrachtet. Husserl diskutiert, inwiefern sie jede intentionale Aktivität in Gang setzen und jeder Urteilsbildung vorausgehen. Aber er untersucht nicht nur die Affektion, sondern auch ihre Auswirkung: Die Weckung von subjektiven Interessen eines Habitualitäten-Ich sowie die vorreflexive Zuwendung, die der manifesten Intentionalität vorausgeht, bilden den Fokus der Untersuchung. Weitere Phänomene der passiven Urteilsbildung wie Widerstreit, Ambivalenz und Hemmung werden als sinnvolle Momente des subjektiven Lebens identifiziert. ${ }^{21}$ Die Assoziation selbst wird explizit als Titel der Intentionalität gewonnen. Sie wird dadurch zu einem transzendental-phänomenologischen Grundbegriff:

Das universale Prinzip der passiven Genesis für die Konstitution aller im aktiven Bilden letztlich vorgegebenen Gegenständlichkeiten trägt den Titel Assoziation. Es ist, wohlgemerkt, ein Titel der Intentionalität $[\ldots] .{ }^{22}$

Diese Konstitutionsstudien stehen nicht für sich allein. Vielmehr müssen sie im Zusammenhang mit den weiterführenden Untersuchungen zur egologischen Subjektivität und ihrer Genese betrachtet werden. Im Jahr 1925, in seinen Vorlesungen zur Phänomenologischen Psychologie, vertieft Husserl in der Auseinandersetzung mit Dilthey die These von der Individualität des Ego als konkrete Subjektivität, die hier auch den Titel Monade erhält. Von Interesse sind dabei die Entwicklungsprozesse der Subjektivität, das heißt die Entstehung und Entfaltung der personalen Einheit. Damit wird zugleich die lebensweltlich relevante Frage nach der Entwicklung des Ich als Subjekt seiner Umwelt intensiv behandelt. In diesem Zusammenhang wird ein näherer Blick auf die subjektive Struktur möglich, die sich als in ihrem Kern intersubjektiv zeigt. ${ }^{23}$ Gerade die intersubjektive Grundbestimmung entscheidet darüber, dass wir nicht Subjekte von isolierten Sonderwelten sind, sondern in Konnex miteinander stehen. Dass wir daher nur

20 Husserl E., Analysen zur passiven Synthesis. Aus Vorlesungs- und Forschungsmanuskripten 19181926, Hua XI, Hrsg. M. Fleischer, Den Haag, Nijhoff, 1966, S. 163.

21 Hua XI, 184.

22 Husserl E., Cartesianische Meditationen und Pariser Vorträge, Hua I, Hrsg. S. Strasser, 1950, 113f.

23 Vgl. z.B. Husserl E., Zur Phänomenologie der Intersubjektivität. Texte aus dem Nachlass. Dritter Teil: 1929-1935. Hua XV, Hrsg. I. Kern, Den Haag, Nijhoff, 1973, $191 f$. 
durch und in Relationen zueinander verständlich gemacht werden können - und zwar als Subjekte der Lebenswelt.

Diese lebensweltlich und intersubjektiv orientierte Weiterführung der phänomenologischen Forschung bedeutet allerdings keinen Bruch in Husserls Gedankengang. Das leitende Ziel der husserlschen Phänomenologie bleibt die Begründung der Objektivität der Erkenntnis, die Begründung der Objektivität unserer Urteile und der Urteilsfähigkeit überhaupt, und dafür ist eine angemessene Deutung der Intersubjektivität unabdingbar. Denn darin liegt die Klärung der Möglichkeit objektiver Erkenntnis einschließlich der logischen Erkenntnis. Dieser Zielsetzung trägt Husserls letztes Werk Erfahrung und Urteil sowie die 1929 veröffentlichte, als Einleitung dazu dienende, Formale und transzendentale Logik Rechnung.

Das genetische Verständnis der Intentionalität ermöglicht in diesen späten Werken einen neuen Zugang zur Frage der intentionalgenetischen Begründung der Logik. Die Urteilstheorie wird neu gedacht, es werden neue Fragestellungen formuliert, die jedoch in Formale und transzendentale Logik noch keine endgültige Lösung finden. Husserl erkennt zwar schon in dem letztgenannten Werk die Notwendigkeit einer Rückführung der Urteilsevidenzen auf ihre vor-prädikativen Bedingungen. ${ }^{24}$ Er sucht nach Evidenzen. Die Begründung der Logik soll durch Ausweis der vor-prädikativen Evidenzen im Prozess der Urteilsbildung erfolgen. Diese Rückführung sollte aber erst in dem posthum publizierten, vom langjährigen Mitarbeiter Husserls, Ludwig Landgrebe, veröffentlichen Werk Erfahrung und Urteil erfolgen. Aber was sind solche vor-prädikativen oder sogar nicht-prädikativen Evidenzen? Sie werden - so Husserl in Erfahrug und Urteil - in lebensweltlichen Erfahrungen gegeben. Die Suche nach diesen primären Evidenzen führt uns zurück auf den Boden der Lebenswelt. Dies soll zwar nicht bedeuten, dass sich die lebensweltliche Erfahrung auf die vor-prädikative beschränken lässt, oder mit ihr gleichgesetzt werden kann. Aber die vor-prädikative Erfahrung ist immer lebensweltlich gebunden und schöpft aus lebensweltlicher Anschaulichkeit. Die lebensweltliche Erfahrung verlangt so keine in Sprache oder in diskursiven Gedanken formulierten exakten Evidenzen. Hier regieren noch gewissermaßen stumme Kenntnisse, Fertigkeiten, Praxen und Geschicke. Hier finden aber auch elementare Prozesse der Typisierung statt, die uns nicht nur die Handhabbarkeit der Welt ermöglichen, sondern auch jeglicher Prädikation und jeglicher Urteilsbildung vorausgehen.

24 Vgl. Husserl E., Formale und transzendentale Logik. Versuch einer Kritik der logischen Vernunft, Hua XVII, Hrsg. P. Janssen, Den Haag, Nijhoff, 1974, 216 ff. 
Der Typus fungiert dabei nicht als Kategorie oder angeborenes Schema wie bei Immanuel Kant. In dieser elementaren Struktur des subjektiven Umgangs mit der Welt wirkt vielmehr eine subjektive Habe, d. h. ein erworbenes Vorwissen, das uns den ersten Zugriff auf Sachen und Situationen im Erfahrungsleben ermöglicht. ${ }^{25}$ Der Typus ist allerdings keine starre Struktur. Er verändert sich vielmehr stets im Laufe der Erfahrung. Diese Veränderung bedeutet keinen dauernden Progress, unser Blick auf die Welt wird nicht immer genauer und präziser; unser Vorwissen kann auch verkümmern, es wird dann undifferenzierter oder enger. Der Typus als subjektive Habe bedeutet zum einen ein nicht expliziertes Erfahrungswissen. Zum anderen wirkt er als eine subjektive Leistungsstruktur. Diese Leistung besteht darin, dass wir nicht wahllos, sondern nach typischen Vorerwartungen zur Weckung unserer Interessen bereit sind.

Die Typen wirken sich in assoziativen Prozessen aus und begründen die Organisation unserer Erwartungsintentionen, die nicht zufällig und nicht a priori zu einem Gegenstand gehören. Vielmehr bilden die Typen sich im Umgang mit den Gegenständen während unseres Erfahrungslebens. Sie ermöglichen so Zuwendungen und Antizipationen im Verlauf konkreter Erfahrungen, die weit vor jeder Explikation des gegenständlichen Sinns liegen und dennoch elementare Bedeutung für die Urteilsbildung haben.

Eine der gewichtigsten Konsequenzen des genetischen Verständnisses der Intentionalität präsentiert der III. Teil von Erfahrung und Urteil. ${ }^{26} \mathrm{Er}$ ist der intentionalen Konstitution von Allgemeingegenständlichkeiten und den Formen des Überhaupt-Urteils gewidmet. Hier wendet sich die Untersuchung den allgemeinsten Gegenständen zu und thematisiert das begreifende Denken. Die Erkenntnis terminiert dabei in reinen bzw. Wesensbegriffen. Die Gewinnung von Wesensbegriffen verlangt die Ablösung von der situativen Verankerung der Erfahrungsgegenstände in ihren empirischen Kontexten. Es geht um die „Loslösung vom Jetzt und Hier der Erfahrungssituation, die in dem Begriff der Objektivität des Denkens beschlossen liegt“27. Dabei findet der Übergang von empirischer bzw. typischer Allgemeinheit zu Wesensallgemeinheit statt. In diesem Zusammenhang kommt dem Begriff des Typus eine neue Relevanz zu. Er fungiert als die konkrete, lebens-

5 Husserl E., Die Lebenswelt. Auslegungen der vorgegebenen Welt und ihrer Konstitution. Texte aus dem Nachlass (1916-1937). Hua XXXIX, Hrsg. R. Sowa, Dordrecht: Springer, 2008, S. 17.

26 Eine systematische Analyse zur Bildung von Allgemeingegenständlichkeiten und der genetischen Urteilsbildung in Erfahrung und Urteil liegt vor in Brudzińska Jagna, „Erfahrung und Urteil“, in Luft Sebastian und Wehrle Maren, Husserl Handbuch. Leben - Werk - Wirkung, Stuttgart, J. B. Metzler, 2017, SS. 104-113.

27 Husserl E., Erfahrung und Urteil. Untersuchungen zur Genealogie der Logik, ausgearbeitet und hg. v. Ludwig Landgrebe, Prag, 1939, S. 384. 
weltlich erworbene Allgemeinheit im Aufbau der eidetischen Erkenntnis. Damit erlangt die Wesenseinsicht eine Rückbindung an die empirischen Evidenzen.

Die eidetische Methode, die auf eidetischer Variation beruht, verlangt eine spezifische Phantasie-Tätigkeit. Wir starten mit einem Ausgangsexempel aus unserer Lebenswelt, das einen faktischen Charakter hat, und wandeln es in der Phantasie beliebig um. Die Variation behält also den Charakter von beliebig usw. ${ }^{28}$ Damit ist natürlich nicht gemeint, dass wir unser Ausgangsexempel unendlich und ausschöpfend abwandeln. Doch ist, dank des Charakters des Phantasiebewusstseins als reine Möglichkeit, eine solche unendliche Variation potentiell möglich. Diese Besonderheit, die auf das Wesen des Phantasiebewusstseins zurückgeht, gewährleistet die Ablösung von kontingenten Vorgaben empirischer Fakten. Wir sind nicht auf Induktionen auf Basis von begrenzten Erfahrungsbeispielen angewiesen. Und doch behalten wir bei der Bildung von auch allgemeinsten Urteilen, den Urteilen überhaupt, den subjektiven Erfahrungsbezug. Diesen Bezug verdanken wir der Leitungsfunktion des Typus bei der Phantasieabwandlung. Der Typus als solcher motiviert unsere assoziativen antizipatorischen Leistungen bei der Phantasie-Variationstätigkeit. Hier spielen Gestaltähnlichkeiten, Verwandtschaften, Erinnerungen und auch Bedürfnisse eine Rolle. Sie alle führen uns zurück auf unsere lebensweltliche Erfahrung und lassen uns aus dieser schöpfen. Damit erschließt Husserl ein neues Verständnis der intentionalen Erkenntnis und der Leistung der Urteilsbildung. Einerseits führt uns diese Leistung in die Bereiche der Wesensallgemeinheit. Andererseits, dank der Leitungsfunktion des Typus, bleibt die Wesenserkenntnis, in denen auch die allgemeinsten Urteile gründen, lebensweltlich verankert.

Jagna Brudzińska ist Professorin für Philosophie am Institut für Philosophie und Soziologie der Polnischen Akademie der Wissenschaften (Warschau), wo sie die Research Group „Philosophische Anthropologie und Sozialphilosophie“ leitet. Sie ist Wissenschaftliche Mitarbeiterin am Husserl-Archiv Köln sowie Koordination der Sektion "Gestalttheorie und Philosophie“ der International Society for Gestalt Theory and its Applications (GTA).

E-mail: jagna.brudzinska@uni-koeln.de

28 Vgl. Ibid., $410 \mathrm{ff}$. 